\title{
Differential Connectivity of Perirhinal and Parahippocampal Cortices within Human Hippocampal Subregions Revealed by High-Resolution Functional Imaging
}

\author{
Laura A. Libby, ${ }^{1}$ Arne D. Ekstrom, ${ }^{1,3}$ J. Daniel Ragland, ${ }^{2}$ and Charan Ranganath ${ }^{1,3}$ \\ Departments of ${ }^{1}$ Psychology and ${ }^{2}$ Psychiatry and Behavioral Sciences and ${ }^{3}$ Center for Neuroscience, University of California, Davis, Davis, CA 95616
}

\begin{abstract}
Numerous studies support the importance of the perirhinal cortex (PRC) and parahippocampal cortex (PHC) in episodic memory. Theories of PRC and PHC function in humans have been informed by neuroanatomical studies of these regions obtained in animal tract-tracing studies, but knowledge of the connectivity of PHC and PRC in humans is limited. To address this issue, we used resting-state functional magnetic resonance imaging to compare the intrinsic functional connectivity profiles associated with the PRC and PHC both across the neocortex and within the subfields of the hippocampus. In Experiment 1, we acquired standard-resolution whole-brain resting-state fMRI data in 15 participants, and in Experiment 2, we acquired high-resolution resting-state fMRI data targeting the hippocampus in an independent sample of 15 participants. Experiment 1 revealed that PRC showed preferential connectivity with the anterior hippocampus, whereas PHC showed preferential connectivity with posterior hippocampus. Experiment 2 indicated that this anterior-posterior functional connectivity dissociation was more evident for subfields CA1 and subiculum than for a combined CA2/ CA3/dentate gyrus region. Finally, whole-brain analyses from Experiment 1 revealed preferential PRC connectivity with an anterior temporal and frontal cortical network, and preferential PHC connectivity with a posterior medial temporal, parietal, and occipital network. These results suggest a framework for refining models of the functional organization of the human medial temporal lobes in which the PRC and PHC are associated with distinct neocortical pathways that, in turn, may differentially interact with regions along the anterior-posterior axis of the hippocampus.
\end{abstract}

\section{Introduction}

The medial temporal lobe (MTL) is a key component of a network of cortical and subcortical areas that are critical for memory (Squire and Zola-Morgan, 1991; Eichenbaum, 2006). These areas include the perirhinal cortex (PRC), parahippocampal cortex (PHC), entorhinal cortex (ERC), and hippocampal formation (HF), which contains subfields cornu ammonis 1-3 (CA1, CA2, CA3), dentate gyrus (DG), and subiculum (Lorente de No, 1934; Duvernoy and Bourgouin, 1998). Lesion and human neuroimaging studies suggest distinct roles for the PRC and PHC in memory formation and retrieval, although it is not clear how best to characterize these roles (Brown and Aggleton, 2001; Davachi, 2006; Eichenbaum et al., 2007; Ekstrom and Bookheimer, 2007; Litman et al., 2009; Ranganath, 2010). Some clues come from tract-tracing studies in rodents and monkeys suggesting that PRC and PHC differ in their anatomical connectivity with sensory and association areas and with hippocampal subfields (Suzuki and Amaral, 1994a,b;

Received July 20, 2011; revised Feb. 23, 2012; accepted March 18, 2012.

Author contributions: L.A.L., A.D.E., J.D.R., and C.R. designed research; L.A.L. performed research; A.D.E. contributed unpublished reagents/analytic tools; L.A.L. analyzed data; L.A.L., A.D.E., J.D.R., and C.R. wrote the paper.

This work was supported by National Institutes of Health Grants R01 MH084895 and R01 MH83734 and the Alfred T. Sloan Foundation. We gratefully acknowledge Dr. Steve Petersen for the helpful suggestions.

Correspondence should be addressed to Laura A. Libby, University of California, Davis, Center for Neuroscience, 1544 Newton Court, Davis, CA 95616. E-mail: Ialibby@ucdavis.edu.

DOI:10.1523/JNEUROSCI.3711-11.2012

Copyright $\odot 2012$ the authors $\quad 0270-6474 / 12 / 326550-11 \$ 15.00 / 0$
Burwell and Amaral, 1998a,b) (see Fig. 1 A,B). However, because anatomical tracer studies are not feasible in humans, it is unknown whether the human PRC and PHC exhibit differential connectivity with higher neocortical regions and with hippocampal subfields.

Functional connectivity analysis of blood oxygenation leveldependent (BOLD) functional magnetic resonance imaging (fMRI) provides a noninvasive means to assess in vivo large-scale connectivity in the human brain (Raichle et al., 2001; Biswal et al., 2010). Coherent low-frequency signal fluctuations recorded during resting-state fMRI reflect broad networks of brain regions linked by direct and indirect anatomical connections (Vincent et al., 2007; Skudlarski et al., 2008; Greicius et al., 2009; Honey et al., 2009; Barnes et al., 2010). To our knowledge, only one previous study (Kahn et al., 2008) has specifically examined intrinsic functional connectivity with human PHC and PRC. The posterior hippocampus and PHC showed significant functional connectivity with retrosplenial cortex and medial and ventrolateral parietal areas, whereas anterior hippocampus and a combined PRC-ERC region showed significant functional connectivity with anterior and ventrolateral temporal cortex. However, the spatial resolution of standard whole-brain fMRI cannot resolve BOLD signal differences across different HF subfields, which have dimensions on the order of millimeters and may contain discrete connectivity patterns not detectable at the larger scale. Newly developed highresolution fMRI techniques centered on the MTL have revealed distinct patterns of activity in different HF subfields during task 

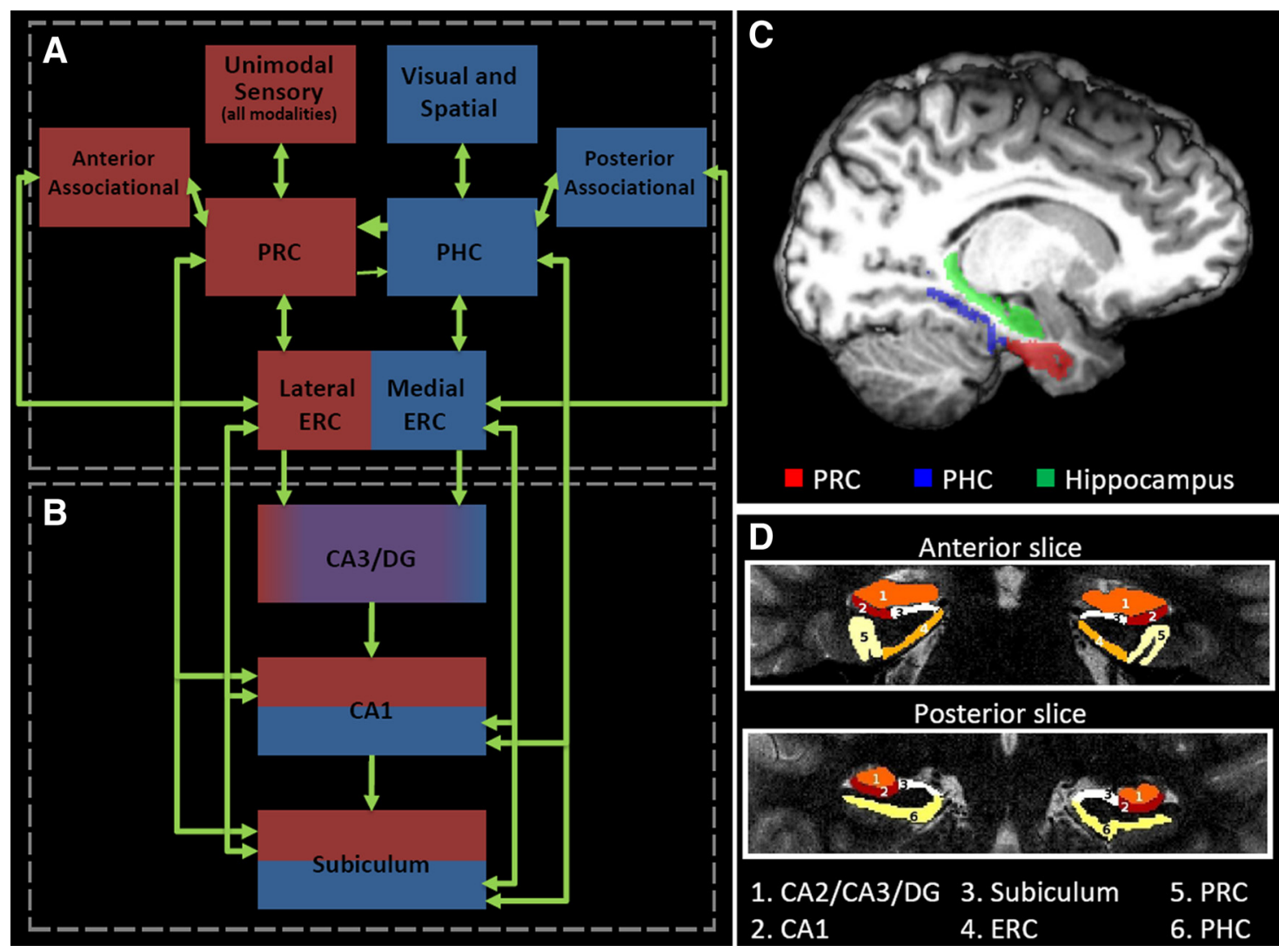

Figure 1. Medial temporal lobe anatomical parcellation. $\boldsymbol{A}, \boldsymbol{B}$, Model of cortical and hippocampal connectivity of PRC and PHC with neocortex $(\boldsymbol{A})$ and hippocampal subfields $(\boldsymbol{B})$ based on animal anterograde and retrograde tracer studies (Burwell, 2000). C, Examples of individually defined ROIs for PRC and PHC for a single subject in Experiment 1.D, Examples of individually defined ROIs for PRC, PHC, ERC, CA1, CA2/CA3/DG, and subiculum in Experiment 2.

(Ekstrom et al., 2009; Suthana et al., 2010; Dudukovic et al., 2011), but this method has not been applied to questions about resting-state functional connectivity in the MTL.

Here, we report results from two experiments examining intrinsic functional networks correlated with signal in PHC and PRC seed regions. In Experiment 1, we used standard-resolution resting-state fMRI to identify distributed whole-brain networks showing preferential functional connectivity with individually anatomically defined PHC and PRC seed regions. In Experiment 2, we used high-resolution resting-state fMRI to characterize functional connectivity between PHC and PRC seeds with anterior versus posterior sections of HF subfields (subiculum, CA1, and CA2/CA3/dentate gyrus).

\section{Materials and Methods \\ Participants}

Two independent samples of 15 young, healthy adults underwent wholebrain (eight females) or high-resolution (eight females) fMRI scanning. Participants were excluded for current use of psychoactive drugs and history of head trauma or neurologic or psychiatric illness. Informed consent was obtained before MRI scanning.

\section{Experiment 1: whole-brain imaging}

Data acquisition and preprocessing. Participants underwent scanning at the UC Davis Imaging Research Center on a 3T Siemens Trio system (Siemens) with an eight-channel phased-array head coil ( $N=15$; two subjects were scanned after a system upgrade to the Total imaging matrix (Tim) acquisition system, but the resulting images were not qualitatively different from images before the upgrade). High-resolution T1-weighted images were acquired using a magnetization-prepared rapid acquisition gradient echo (MPRAGE) pulse sequence (matrix size, $256 \times 256$; 192 slices; voxel size, $1 \times 1 \times 1 \mathrm{~mm}$ ). Images sensitive to BOLD contrast were acquired using a gradient echo planar imaging (EPI) sequence (210 time points; matrix size, $64 \times 64$; TR, $2000 \mathrm{~ms}$; TE, $25 \mathrm{~ms}$; FOV, 220; 34 slices, interleaved; voxel size, $3.4375 \times 3.4375 \times 3.4 \mathrm{~mm}$ ) for 7 min while participants rested with eyes open and lights on a low setting.

The FMRI Expert Analysis Tool in the FMRIB Software Library (FSL version 4.1; www.fmrib.ox.ac.uk/fsl) was used for fMRI analysis. Brain volumes were extracted from full-head functional and structural images. Functional images were motion corrected and bandpass filtered for frequencies of $0.01-0.1 \mathrm{~Hz}$. To prepare volumes for time course extraction, functional images were registered to each participant's MPRAGE image using a rigid-body transformation $(\mathrm{df}=6)$ using FMRIB's Linear Image Registration Tool (FLIRT) (Jenkinson and Smith, 2001; Jenkinson et al., 2002).

Demarcations of anatomical regions of interest. Boundaries between PHC and PRC were defined manually on individual participant native-space MPRAGE images in FSLView using criteria outlined by Insausti et al. (1998), Duvernoy and Bourgouin (1998) and Zeineh et al. (2001). PHC regions of interest (ROIs) extended from the first slice containing the body of the hippocampus to the slice halfway through the lateral ventricles in the anterior-posterior direction, and from the collateral sulcal fundus to the most medial vertex of the parahippocampal gyrus in the lateral-medial direction. PRC ROIs extended from the most anterior slice of the collateral sulcus to the last slice containing the hippocampal head in the anterior-posterior direction and across both banks of the collateral sulcus in the lateral-medial direction. PHC and PRC ROIs for right and left hemispheres were created separately by manual tracing of gray matter within these demarcations (Fig. 1C). To reduce variability in EPI time series due to susceptibility artifact, thresholds were applied to ROIs to exclude voxels with a mean signal in the intensitynormalized (mean, 1000) EPI time series below 3000, corresponding approximately to a signal-to-noise (SNR) ratio of 20 (Firbank et al., 
2000). Thresholding led to the rejection of no more than $5 \%$ of voxels in any ROI. These thresholded ROIs were used as seed regions in ensuing functional connectivity analyses. White matter (WM) and CSF probability maps were generated using FMRIB's Automated Segmentation Tool and thresholded at $p$ (volume) $=1$ to create WM and CSF ROIs.

Functional connectivity analysis. Mean time series for all voxels within left and right seed ROIs as well as WM and CSF ROIs were extracted from individually registered preprocessed functional images and mean centered. Beyond initial preprocessing, functional images then underwent smoothing with a $5 \mathrm{~mm}$ full-width at half-maximum Gaussian kernel. Separate analyses were conducted using a general linear model (GLM) to estimate regions that showed significant functional connectivity with the PRC and PHC. These analyses were conducted separately because the functional connectivity profiles of closely interconnected regions (such as PRC and PHC) may be highly overlapping. Given that the GLM estimates unique contributions of each regressor of interest, including both PRC and PHC time courses in the same GLM could artificially exaggerate differences between the connectivity profiles of these regions. Therefore, four separate GLM analyses were run, one for each seed ROI (right or left PHC or PRC).

In each GLM, the mean-centered time series for each seed ROI was included as a covariate of interest. To control for nonneuronal sources of variance, such as heart rate and respiration, WM and CSF mean-centered time series were included as covariates of no interest (Wise et al., 2004; Birn et al., 2006). Functional connectivity results maps were registered in a two-step process, first to individual subject MPRAGE images $(\mathrm{df}=6)$ and then to the Montreal Neurological Institute (MNI) 152 brain template $(\mathrm{df}=12)$ to prepare individual data for group-

level analysis. Finally, voxelwise group-level analyses were performed. As described in detail below, the group analyses were either one-sample or paired-sample $t$ tests performed on parameter estimates derived from the single-subject GLM analyses. All resulting group $t$ statistic images were converted to (Gaussianized) $z$ statistic images and thresholded using clusters determined by $z>2.3$ and a corrected cluster significance threshold of $p<0.05$ (Worsley et al., 1996).

Group analyses were conducted in three steps (Fig. 2A). (1) For single-seed connectivity maps, four one-sample $t$ tests were conducted, each assessing connectivity with a separate seed ROI. (2) For within-seed hemispheric asymmetry maps, paired-sample $t$ tests were performed, each comparing functional connectivity maps associated with seeds in right and left hemispheres. These $t$ tests were conducted separately for seeds in PRC and PHC and yielded voxel maps reflecting hemispheric asymmetry of the functional connectivity profile of each seed ROI. (3) For maps of differential connectivity with PRC and PHC, two voxelwise paired-sample $t$ tests were performed, each contrasting parameter estimates extracted from the GLM assessing functional connectivity with either the left or right PRC seed against parameter estimates extracted from the GLM assessing connectivity with the ipsilateral PHC seed. Because resulting voxel clusters were highly extensive and connected, to describe the full pattern of results, regional local maxima were identified by locating the 60 peak local maxima within each cluster (Worsley et al., 1996) and then selecting from these the greatest maximum for each anatomical region covered by the cluster.
Individual Level GLMs

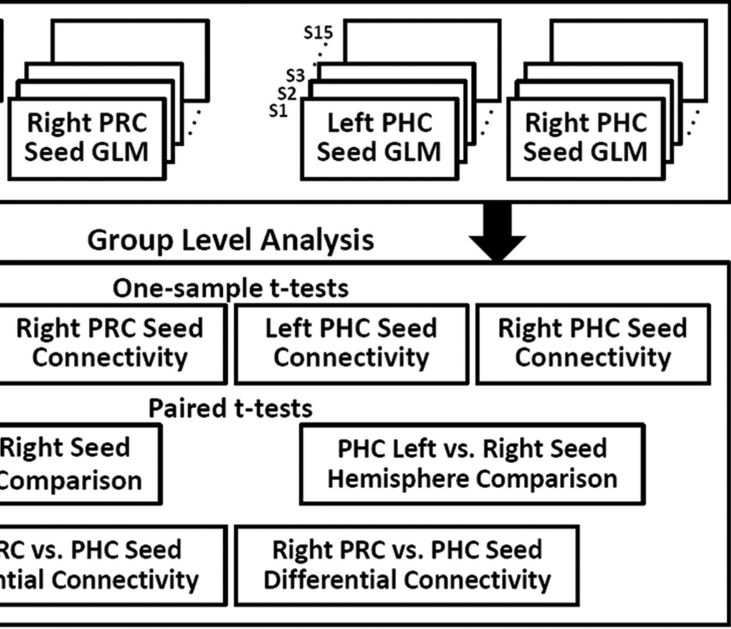

Individual Level GLMs
Left PRC vs. PHC Seed Right PRC vs. PHC Seed

Differential Connectivity

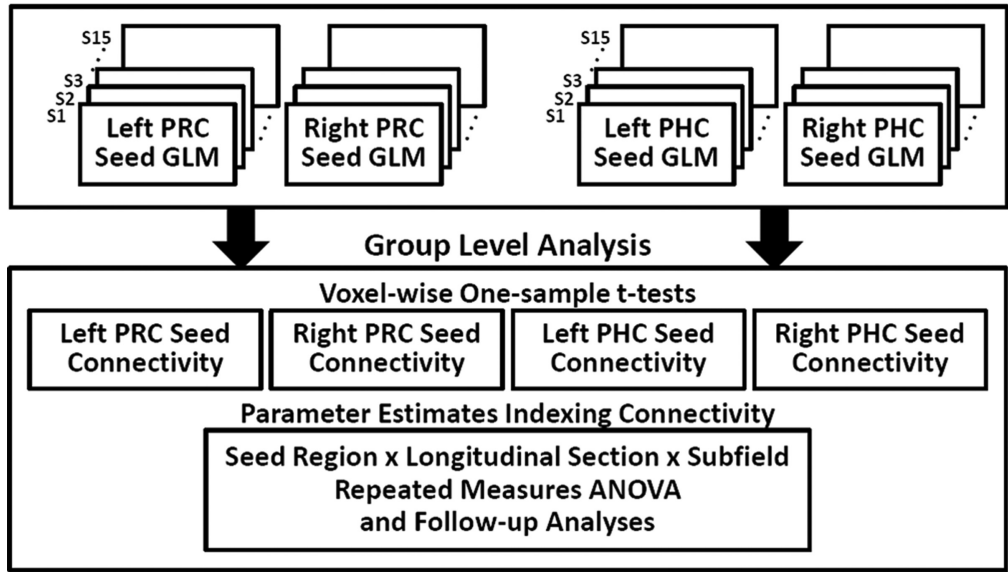

\section{B Experiment 2}

Figure 2. $\quad \boldsymbol{A}, \boldsymbol{B}$, Summary of functional connectivity analysis procedures for Experiment $1(\boldsymbol{A})$ and Experiment $2(\boldsymbol{B})$.

\section{Experiment 2: high-resolution imaging}

Data acquisition and preprocessing. Participants were scanned at the UC Davis Imaging Research Center on a 3T Siemens Tim Trio system with a 32-channel phased-array head coil. Sagittal T1-weighted images were acquired using an MPRAGE sequence (matrix size, $256 \times 256$; 208 slices; voxel size, $1 \times 1 \times 1 \mathrm{~mm}$ ) to localize the hippocampal formation for alignment of high-resolution images. High-resolution T2-weighted structural images were acquired in an oblique coronal plane perpendicular to the longitudinal axis of the hippocampus using a spin-echo sequence (matrix size, $200 \times 161 \mathrm{~mm}$; TR, $4200 \mathrm{~ms}$; TE, $106 \mathrm{~ms}$; FOV, $20 \mathrm{~cm}$; 28 slices, interleaved; voxel size, $0.4 \times 0.4 \times 1.9 \mathrm{~mm}$ ). Images sensitive to BOLD contrast were acquired using a high-resolution gradient-echo EPI sequence (200 time points; matrix size, $128 \times 128$ mm; TR, 3000 ms; TE, 33 ms; FOV, 192 mm; 40 slices, interleaved; voxel size, $1.5 \times 1.5 \times 1.9 \mathrm{~mm}$ ) for $10 \mathrm{~min}$ at rest with eyes open and lights off in the same plane centered on the hippocampus and perpendicular to the longitudinal axis of the hippocampus. Coplanar matched-bandwidth high-resolution gradient echo EPI sequences (200 time points; matrix size, $128 \times 128 \mathrm{~mm}$; TR, $3000 \mathrm{~ms}$; TE, $33 \mathrm{~ms}$; FOV, $192 \mathrm{~mm}$; 40 slices, interleaved; voxel size, $1.5 \times 1.5 \times 1.9 \mathrm{~mm}$ ) were also acquired for registration of functional scans to structural images (Ekstrom et al., 2009). Preprocessing procedures for highresolution fMRI data were identical to methods applied to wholebrain images in Experiment 1, described above.

Demarcations of anatomical regions of interest. Boundaries between PRC, ERC, PHC, subiculum, CA1, and a CA2/CA3/DG region were de- 
fined manually on individual participant high-resolution structural images in FSLView according to Insausti et al. (1998), Duvernoy and Bourgouin (1998), and Zeineh et al. (2001) (Fig. 1D). As in Experiment 1 , right and left PRC and PHC ROIs were thresholded to remove voxels with low functional signal (normalized intensity of $<2500$, corresponding to an SNR of 65) (Firbank et al., 2000), and these thresholded masks were used as seed ROIs in ensuing functional connectivity analyses. For analysis of differences along the longitudinal axis of the hippocampus, subiculum, CA1, and CA2/CA3/DG ROIs were each additionally divided into head (slices 6-12), body (slices 13-18), and tail (slices 19-25) sections based on anatomical landmarks (Duvernoy and Bourgouin, 1998). WM and CSF ROIs were created according to the procedure described for Experiment 1.

Functional connectivity analysis. Functional connectivity analyses in Experiment 2 for right and left PRC and PHC seeds were identical to analyses in Experiment 1, with the following exceptions. Functional connectivity volumes were registered into individual-subject structural space in FLIRT via a two-step process, first to coplanar matchedbandwidth images $(\mathrm{df}=6)$ and then to high-resolution $\mathrm{T} 2$ images $(\mathrm{df}=$ 6). To optimize ROI registration across individuals, linear registration $(\mathrm{df}=12)$ was performed in FLIRT to align individually registered functional connectivity volumes with a single participant's high-resolution structural image for group analysis. Then, resulting group-registered contrast images underwent nonlinear diffeomorphic registration (smoothness constraint, two voxels) within the MTL using the ROIbased Alignment procedure (Yassa and Stark 2009), providing a single group MTL image reflective of individual nonlinear anatomical variation. BOLD signal loss in the ERC due to susceptibility artifact precluded detailed analysis of functional connectivity in this area.

To determine the topographic patterns of functional connectivity within the HF associated with PHC and PRC, group-level functional connectivity profiles for each seed region were calculated with voxelwise one-sample $t$ tests (Fig. $2 B$ ). Resulting $t$ statistic images were converted to (Gaussianized) $z$ statistic images, masked to exclude voxels outside of the MTL, and thresholded at $z>2.3$ and a corrected cluster significance threshold of $p<0.05$ (Worsley et al., 1996).

To examine the distribution of PRC and PHC functional connectivity along the anterior-posterior axis of the HF, we plotted mean parameter estimates indexing functional connectivity obtained from normalized individual-level PRC and PHC connectivity analyses (voxelwise onesample $t$ tests) for each slice of right and left subiculum, CA1, and CA2/ CA3/DG. To assess the differential topography of PRC versus PHC functional connectivity along the anterior-posterior axis by subfield, we extracted parameter estimates indexing connectivity with PRC and PHC (from voxelwise one-sample $t$ tests) averaged across all voxels in either head, body, or tail segments of each subfield. These PRC and PHC connectivity estimates were submitted to repeated-measures ANOVA with seed ROI (PHC vs PRC), seed hemisphere, target ROI (CA2/CA3/DG vs CA1 vs subiculum), target hemisphere, and longitudinal section (head vs body vs tail segment of each subfield) as factors, using the Huynh-Feldt correction for nonsphericity (Huynh and Feldt, 1976; Lecoutre, 1991).

To characterize effects detected in the above ANOVA, a set of planned comparisons were performed at the level of each subfield at each longitudinal section (e.g., the effect of seed ROI within CA1 head). Initial comparisons of PRC and PHC connectivity indicated no significant difference in the head of CA2/CA3/DG, but slice-by-slice extractions suggested that differential connectivity may be stronger in the anterior three slices of CA2/CA3/DG compared to other slices in the head section. Therefore, we repeated the relevant comparison, substituting connectivity estimates extracted from only the most anterior three slices of CA2/ CA3/DG for estimates from the entire head segment of this subregion. Additionally, because planned comparisons suggested differential PHC connectivity in the tail segments of all three subfields, a further set of comparisons determined whether differential PHC connectivity differed in magnitude between the tail segments of CA2/CA3/DG, CA1, and subiculum.

\section{Results}

\section{Experiment 1: whole-brain imaging}

Our first analyses focused on separately characterizing the intrinsic connectivity profiles of the PHC and PRC. Figure 3 shows the functional connectivity maps for left and right hemisphere seed ROIs in the PRC and PHC. As seen in the figure, for both PRC and PHC, functional connectivity maps for seeds in right and left hemispheres were visually similar to one another. In general, the PRC exhibited connectivity with a network of regions in the anterior temporal and the ventrolateral prefrontal cortices, with considerable similarity between seeds in right and left hemispheres. The PHC exhibited connectivity with a network of regions that included areas in the posterior midline (retrosplenial, posterior cingulate, and precuneus) and occipital cortex that was similar for right and left hemisphere seeds. Additionally, both PRC and PHC showed a high degree of connectivity across the entire MTL.

The second set of analyses compared the functional connectivity of seeds in right and left hemispheres separately for PRC and PHC (Fig. 3, bottom row). Compared with seeds in the contralateral hemisphere, all seeds exhibited preferential connectivity with select voxels in the MTL ipsilateral to the seed, including PRC, PHC, and anterior and posterior HF, and with select voxels in ipsilateral cortex outside of the MTL. For connectivity with PRC, significant asymmetries were observed in lateral temporal and medial and lateral prefrontal cortex. For connectivity with PHC, significant asymmetries were observed in medial and lateral parietal and lateral prefrontal cortices. Critically, these analyses revealed (1) a small number of voxels relative to one-sample $t$ test results and (2) no significant voxels contralateral to any seed region, suggesting that, for both $\mathrm{PRC}$ and $\mathrm{PHC}$, connectivity associated with seeds in one hemisphere replicated connectivity associated with seeds in the other hemisphere.

Our next analyses focused on contrasts between whole-brain functional connectivity maps for seeds in PHC and PRC (Fig. 4). To test directly for relative differences in whole-brain functional connectivity with these two seed regions, we statistically contrasted functional connectivity maps associated with PHC and PRC seed regions from the same hemisphere. Paired $t$ tests revealed distinct, bilateral pathways that differentially connected with the PRC and PHC seed regions. PRC showed relatively greater functional connectivity with an anterior network that included anterior fusiform cortex; posterior lateral and inferior temporal cortices; temporoparietal cortex; precentral and postcentral gyri; orbitofrontal cortex; dorsolateral, ventrolateral, and dorsomedial prefrontal cortices; and anterior HF. PHC showed relatively greater functional connectivity with a posterior network that included medial and lateral occipital cortex, precuneus, posterior cingulate cortex, pulvinar, retrosplenial cortex, and posterior HF. Table 1 provides local maxima defined for anatomical regions within each network.

\section{Experiment 2: high-resolution imaging}

Results from Experiment 1 indicated that the PHC showed relatively stronger connectivity with the posterior hippocampus, and that PRC showed relatively stronger connectivity with the posterior hippocampus. In Experiment 2, we used high-resolution fMRI centered on the MTL to investigate intrinsic functional connectivity between PHC and PRC seeds and subfields of the HF (Fig. 5). To characterize the topography of PRC and PHC functional connectivity along the longitudinal axis of the HF, we plotted mean parameter estimates indexing connectivity with the $\mathrm{PRC}$ and PHC seeds (from one-sample $t$ tests) for each slice in the 
six HF ROIs (right and left subiculum, CA1, and CA2/CA3/DG). As shown in Figure $6 A$, the results revealed different distributions of PRC and PHC functional connectivity across the length of the HF, and the extent of the connectivity differences along the longitudinal axis appeared to differ across the three subfields.

To quantify these differences, we segmented each HF subfield into anatomically based head, body, and tail sections (Fig. 6B) and extracted parameter estimates indexing mean PRC and PHC functional connectivity (from one-sample $t$ tests) across all voxels in each of these sections for each individual. A repeatedmeasures ANOVA of PRC and PHC connectivity estimates from each longitudinal section of CA2/CA3/DG, CA1, and subiculum revealed significant two-way interactions of seed region by longitudinal section $\left(F_{(2,28)}=101.76, p<0.001, \varepsilon_{\mathrm{H}-\mathrm{F}}\right.$ $=0.64)$ and seed region by subfield $\left(F_{(2,28)}=21.33, p<0.001, \varepsilon_{\mathrm{H}-\mathrm{F}}=1.07\right)$, and, most importantly, a significant three-way interaction of seed region by longitudinal section by subfield $\left(F_{(4,56)}=\right.$ $\left.8.92, p>0.001, \varepsilon_{\mathrm{H}-\mathrm{F}}=0.74\right)$. The significant three-way interaction confirmed that differences in the connectivity of PRC and PHC along the anterior-posterior hippocampal axis differed across subfields.

Follow-up analyses to break down this three-way interaction revealed that PRC connectivity was significantly greater than PHC connectivity in the head segment of $\operatorname{CA1}\left(F_{(1,14)}=55.99, p<0.001\right)$ and subiculum $\left(F_{(1,14)}=30.74, p<0.001\right)$ and the body segment of CA1 $\left(F_{(1,14)}=6.31, p<\right.$ $0.05)$. In contrast, $\mathrm{PHC}$ connectivity was significantly greater than PRC connectivity in the body segment of CA2/CA3/DG $\left(F_{(1,14)}=38.42, p<0.001\right)$ and subiculum $\left(F_{(1,14)}=5.90, p<0.05\right)$ and the tail segments ofCA2/CA3/DG $\left(F_{(1,14)}=45.45, p<\right.$ $0.001), \operatorname{CA} 1\left(F_{(1,14)}=5.86, p<0.05\right)$, and subiculum $\left(F_{(1,14)}=64.09, p<0.001\right)$. PRC and $\mathrm{PHC}$ connectivity estimates were not significantly different in CA2/CA3/DG head (Fig. 6C).

The comparisons described above indicated that, relative to PHC, PRC showed significantly higher connectivity with the head of the hippocampus in the subiculum and CA1. Although functional connectivity estimates for PRC and PHC seeds did not differ significantly in the head of CA2/CA3/ DG, slice-by-slice extractions suggested that differential connectivity may be present in the most anterior slices of this subfield (Fig. 6A). Additional analysis indeed revealed that PRC connectivity was significantly greater than $\mathrm{PHC}$ connectivity within the most anterior three slices of CA2/CA3/DG $\left(F_{(1,14)}=4.53, p<0.05\right)$.

With regard to the tail of the hippocampus, our analyses revealed that each subfield tail showed greater connectivity with the PHC than with the PRC, although the magnitude of this dif-
Parahippocampal Cortex Seeds
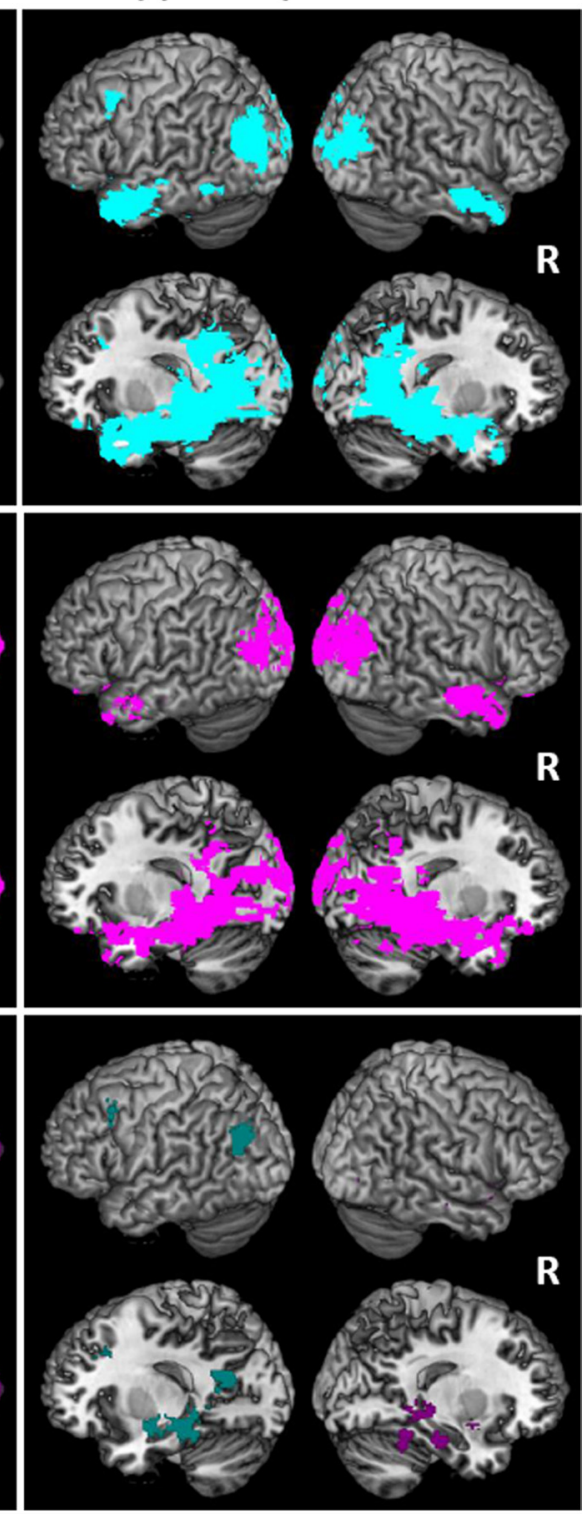

LH $>$ RH Seed $\quad$ RH $>$ LH Seed

Figure 3. Whole brain analysis of functional connectivity with PRC and PHC. One-sample $t$ test (top two rows) and left (LH) versus right hemisphere $(\mathrm{RH})$ seed paired $t$ test (bottom row) whole-brain functional connectivity results $\left(z>2.3, p_{\text {cluster }}<0.05\right)$ mapped separately for seeds in PRC and PHC. Cyan, LH seed connectivity only; pink, RH seed connectivity only; teal, LH seed greater than RH seed connectivity; purple, RH seed greater than LH seed connectivity.

ference appeared to vary across subfields. An additional repeated-measures (subfield by seed region) ANOVA restricted to tail segments revealed a significant two-way interaction $\left(F_{(2,28)}=12.26, p<0.001, \varepsilon_{\mathrm{H}-\mathrm{F}}=1.00\right)$, and follow-up tests revealed that preferential $\mathrm{PHC}$ connectivity was greater in subiculum tail compared to CA2/CA3/DG tail $\left(F_{(1,14)}=\right.$ $5.72, p<0.05)$, and greater in CA2/CA3/DG tail compared to CA1 tail $\left(F_{(1,14)}=7.58, p<0.01\right)$.

In summary, the results of Experiment 2 converged with those of Experiment 1 to suggest a gradient of functional connectivity with the PHC and PRC along the extent of the hippocampus. The high-resolution data from Experiment 2 further indicated that this pattern differed across hippocampal subregions. Whereas PHC showed preferential connectivity with tail segments of all 


\section{A Left Hemisphere Seeds}

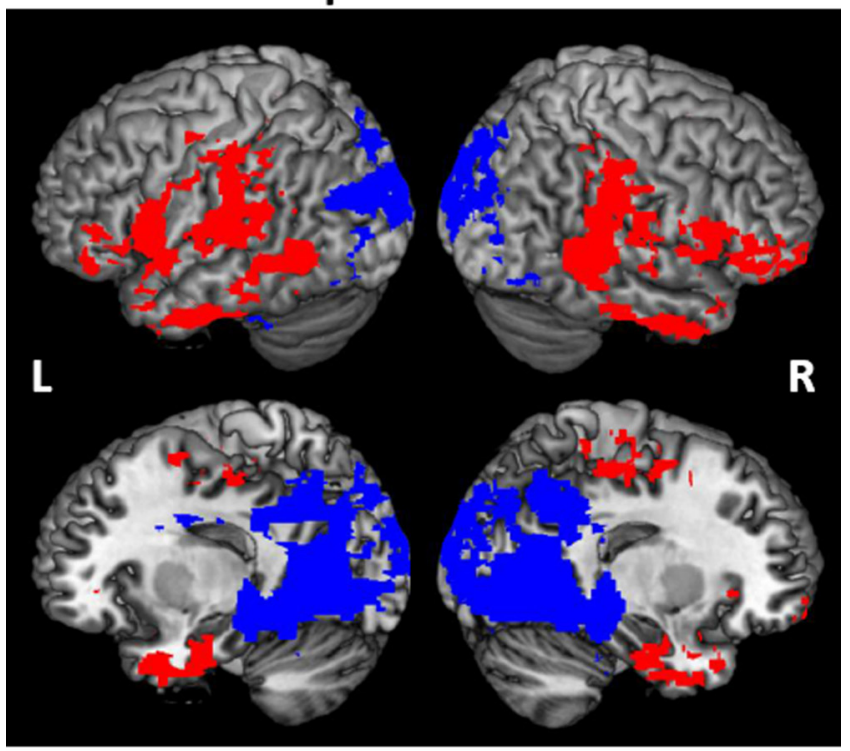

\section{B Right Hemisphere Seeds}

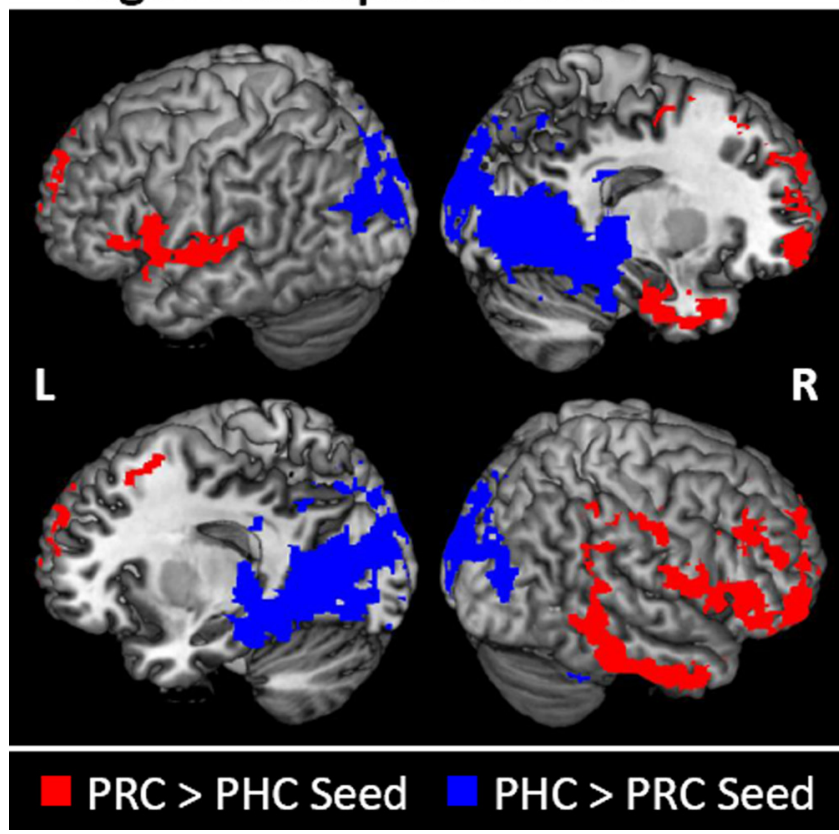

Figure 4. Comparison of cortical connectivity patterns between PRC and PHC seed regions. $\boldsymbol{A}, \boldsymbol{B}$, Paired $t$ test whole-brain functional connectivity results $\left(z>2.3, p_{\text {cluster }}<0.05\right)$ mapped separately for seeds in the left hemisphere $(\boldsymbol{A})$ and right hemisphere $(\boldsymbol{B})$. Red, PRC seed greater than PHC seed connectivity; blue, PHC seed greater than PRC seed connectivity.

three subfields, but most strongly for subiculum, PRC showed only preferential connectivity with head segments of CA1 and subiculum.

\section{Consideration of alternate accounts}

Although functional connectivity measures can be influenced by nonneuronal sources of BOLD signal covariance (van den Heuvel and Hulshoff Pol, 2010), these factors cannot sufficiently explain the current findings. For instance, if the anterior and posterior MTL were vascularized by two separate systems of blood vessels, relative differences in blood flow could potentially yield preferential BOLD correlations between signals in PRC and anterior hippocampus and $\mathrm{PHC}$ and posterior hippocampus. However, such an anterior-posterior vascular division within both hippocampus and parahippocampal gyrus is not supported by cerebrovascular studies (Marinković et al., 1991, 1992; Erdem et al., 1993; Duvernoy and Bourgouin, 1998; Huther et al., 1998). Most commonly, PRC, PHC, and hippocampus are vascularized by arteries arising from a combination of the anterior choroidal and posterior cerebral arteries. It is therefore improbable that the robust and reliable (found in both Experiment 1 and Experiment 2) anterior-posterior functional connectivity dissociation within the hippocampus arose due to differences in vascular origin between anterior and posterior MTL.

Another factor that can spuriously influence resting-state functional connectivity estimates is head motion, which has the effect of inflating correlations between a seed voxel and proximal voxels (Power et al., 2011). Therefore, when the connectivity patterns of two seeds are compared within the same individual, as in the current study, motion artifact could theoretically induce preferential connectivity between each seed and the geometrically closest voxels. However, in both Experiments 1 and 2, it was not exclusively the case that a brain region showed relatively stronger functional connectivity with the seed region to which it was geometrically closest. For instance, in Experiment 1, the temporoparietal junction was preferentially functionally connected with PRC, but geometrically closer to PHC, and in Experiment 2, the body segment of CA1 was preferentially connected with PRC, but geometrically closer to $\mathrm{PHC}$. This disconnect between proximity and connectivity strength argues against a motion artifact explanation, and thus simultaneous neural activity rather than simple spatial proximity is the more parsimonious explanation of the current results.

\section{Discussion}

In the current study, we compared functional connectivity profiles of human PRC and PHC using both whole-brain imaging and high-resolution imaging centered on the MTL. Experiment 1 revealed that $\mathrm{PRC}$ showed preferential connectivity with anterior hippocampus, whereas PHC showed preferential connectivity with posterior hippocampus. High-resolution fMRI results from Experiment 2 indicated that CA1 and subiculum, but not CA2/ CA3/DG, showed the expected pattern of differential PRC connectivity across anterior hippocampus and differential PHC connectivity across posterior hippocampus, and that this anterior-posterior connectivity gradient was strongest in subiculum. Finally, whole-brain analyses from Experiment 1 revealed preferential PRC connectivity with an anterior temporal and frontal cortical network, and preferential PHC connectivity with a posterior medial temporal, parietal, and occipital network.

Functional connectivity within the MTL

Findings from Experiment 1 converged with those of Kahn et al. (2008) in showing differential connectivity of the PRC and PHC with the anterior and posterior hippocampus. Experiment 2 expanded our understanding of human corticohippocampal connectivity by allowing for separate investigation of connectivity with different hippocampal subfields. Studies in animal models suggest that subfields CA2/CA3/DG, CA1, and subiculum have indirect reciprocal connections with the $\mathrm{PRC}$ and $\mathrm{PHC}$ via the entorhinal cortex (Fig. $1 A$ ) that terminate broadly along the anterior-posterior axis of the hippocampus (Lavenex and Amaral, 2000; Kerr et al., 2007). However, in rats, additional, direct connections from PRC have been found to terminate primarily in ventral (analogous to anterior HF in primates) CA1 and subiculum, and direct projections 


\begin{tabular}{|c|c|c|c|c|c|c|c|c|}
\hline & \multicolumn{4}{|c|}{ LH voxels } & \multicolumn{4}{|c|}{ RH voxels } \\
\hline & \multicolumn{3}{|c|}{ Local maximum (MNI) } & \multirow[t]{2}{*}{ zscore } & \multicolumn{3}{|c|}{ Local maximum (MNI) } & \multirow[t]{2}{*}{ zscore } \\
\hline & $x$ & $y$ & $z$ & & $x$ & $y$ & $z$ & \\
\hline Angular gyrus (BA 39) & -40 & -48 & 50 & $3.92^{\dagger}$ & 52 & -48 & 28 & $3.88^{\diamond}$ \\
\hline Anterior hippocampus & -24 & -12 & -30 & $4.51^{\dagger}$ & 22 & -4 & -26 & $3.31^{*}$ \\
\hline Anterior inferior temporal gyrus (BA 20) & -56 & -32 & -24 & $5.07^{\dagger}$ & 64 & -14 & -28 & $4.2^{*}$ \\
\hline Inferior frontal gyrus (BA 46) & -58 & 20 & -2 & $4.15^{\dagger}$ & 54 & 38 & 2 & $3.66^{\diamond}$ \\
\hline Lateral postcentral gyrus (BA 40) & -44 & -36 & 52 & $4.09^{\dagger}$ & 42 & -46 & 42 & $3.24^{\diamond}$ \\
\hline Lateral precentral gyrus (BA4) & -34 & -20 & 54 & $3.86^{\dagger}$ & & & & \\
\hline Lateral precentral gyrus (BA6) & -52 & 4 & 12 & $4.62^{\dagger}$ & 60 & 4 & 4 & $4.47^{\diamond}$ \\
\hline Orbitofrontal cortex (BA 11/47) & -16 & 24 & -20 & $4.25^{\ddagger}$ & 24 & 12 & -24 & $3.85^{*}$ \\
\hline Posterior middle temporal gyrus (BA 21) & -64 & -36 & -10 & $4.15^{\dagger}$ & 54 & -2 & -32 & $3.26^{\diamond}$ \\
\hline Ventrolateral prefrontal cortex (BA 44/45) & -54 & 12 & 16 & $4.83^{\dagger}$ & 54 & 18 & 12 & $4.17^{\diamond}$ \\
\hline \multicolumn{9}{|l|}{$\mathrm{RPRC}>\mathrm{RPHC}$} \\
\hline Angular gyrus (BA 39) & & & & & 48 & -36 & 44 & $3.6^{\dagger}$ \\
\hline Anterior hippocampus & & & & & 28 & -12 & -24 & $3.47^{\dagger}$ \\
\hline Anterior inferior temporal gyrus (BA 20) & & & & & 54 & -12 & -30 & $4.54^{\dagger}$ \\
\hline Dorsolateral prefrontal cortex (BA 9) & -24 & 60 & 24 & $4.22^{\ddagger}$ & 18 & 58 & 24 & $3.6^{\dagger}$ \\
\hline Dorsomedial prefrontal cortex (BA 8) & -2 & -60 & 34 & $3.62^{\ddagger}$ & & & & \\
\hline Frontal polar cortex (BA 10) & & & & & 40 & 60 & -2 & $3.79^{\dagger}$ \\
\hline Fusiform cortex (BA 20) & & & & & 54 & -12 & -30 & $4.54^{\dagger}$ \\
\hline Inferior frontal gyrus (BA 47) & & & & & 56 & 26 & -8 & $4.5^{\dagger}$ \\
\hline Lateral precentral gyrus (BA 6) & & & & & 54 & 4 & 10 & $4.07^{\dagger}$ \\
\hline Medial prefrontal cortex (BA 8) & -2 & 60 & 34 & $3.62^{\ddagger}$ & & & & \\
\hline \multicolumn{9}{|l|}{$\mathrm{LPHC}>\mathrm{LPRC}$} \\
\hline Lateral occipital cortex (BA 19) & -38 & -82 & 28 & $4.55^{\dagger}$ & 16 & -98 & 20 & $4.15^{\dagger}$ \\
\hline Medial posterior occipital cortex (BA 18) & -2 & -78 & -2 & $4.9^{\dagger}$ & 6 & -58 & 14 & $4.43^{\dagger}$ \\
\hline Occipital pole (BA 17) & -8 & -58 & -30 & $4.38^{\dagger}$ & 8 & -102 & 16 & $4.45^{\dagger}$ \\
\hline Parahippocampal cortex & -14 & -50 & -6 & $4.72^{\dagger}$ & 18 & -46 & -4 & $5.01^{\dagger}$ \\
\hline Posterior cingulate cortex (BA 29) & -8 & -50 & 14 & $4.74^{\dagger}$ & 8 & -46 & 16 & $4.31^{\dagger}$ \\
\hline Posterior hippocampus & -20 & -30 & -8 & $4.66^{\dagger}$ & 20 & -30 & -6 & $5.35^{\dagger}$ \\
\hline Posterior thalamus & -20 & -32 & -2 & $3.82^{\dagger}$ & 22 & -32 & 2 & $3.92^{\dagger}$ \\
\hline Precuneous (BA 7) & -14 & -60 & 18 & $4.59^{\dagger}$ & 18 & -68 & 24 & $4.89^{\dagger}$ \\
\hline Retrosplenial cortex (BA 30) & -8 & -50 & 14 & $4.74^{\dagger}$ & 8 & -58 & 4 & $4.81^{\dagger}$ \\
\hline \multicolumn{9}{|l|}{$\mathrm{RPHC}>\mathrm{RPRC}$} \\
\hline Lateral occipital cortex (BA 19) & -40 & -86 & 24 & $4.14^{\dagger}$ & & & & \\
\hline Medial posterior occipital cortex (BA 18) & & & & & 14 & -72 & 8 & $4.21^{\dagger}$ \\
\hline Occipital pole (BA 17) & -16 & -96 & 22 & $4.28^{\dagger}$ & 14 & -88 & 4 & $4.22^{\dagger}$ \\
\hline Parahippocampal cortex & -12 & -42 & -8 & $4.42^{\dagger}$ & 22 & -32 & -8 & $5.56^{\dagger}$ \\
\hline Posterior cingulate cortex (BA 29) & -4 & -46 & 4 & $4.33^{\dagger}$ & 10 & -44 & 10 & $4.13^{\dagger}$ \\
\hline Posterior hippocampus & -20 & -30 & -2 & $4.88^{\dagger}$ & 18 & -36 & 0 & $5.92^{\dagger}$ \\
\hline Posterior thalamus & -20 & -34 & 0 & $4.88^{\dagger}$ & 22 & -30 & 6 & $5.03^{\dagger}$ \\
\hline Precuneus (BA 7) & -12 & -50 & 40 & $3.26^{\ddagger}$ & 18 & -52 & 36 & $3.61^{\ddagger}$ \\
\hline Retrosplenial cortex (BA 30) & -16 & -52 & -4 & $4.26^{\dagger}$ & 22 & -46 & 0 & $5.3^{\dagger}$ \\
\hline
\end{tabular}

LH, Left hemisphere; $\mathrm{RH}$, right hemisphere. Local maxima were defined for anatomical regions included in each cluster. Symbols indicate the extent of the cluster from which each local maximum was obtained: $\mathrm{LPRC}>\mathrm{LPHC}{ }^{\dagger} 250.42 \mathrm{~cm}{ }^{3}$ ${ }^{\ddagger} 28.26 \mathrm{~cm}^{3},{ }^{\diamond} 86.04 \mathrm{~cm}^{3},{ }^{*} 35.60 \mathrm{~cm}^{3} ; \mathrm{RPRC}>\mathrm{RPHC},{ }^{\dagger} 199.84 \mathrm{~cm}^{3},{ }^{\ddagger} 25.62 \mathrm{~cm}^{3} ; \mathrm{LPHC}>\mathrm{LPRC},{ }^{\dagger} 444.72 \mathrm{~cm}^{3} ; \mathrm{RPHC}>\mathrm{RPRC},{ }^{\dagger} 433.26 \mathrm{~cm}^{3},{ }^{\ddagger} 9.66 \mathrm{~cm}^{3}$.

from PHC terminate primarily in dorsal (analogous to posterior HF in primates) CA1 and subiculum, whereas CA2/CA3/DG does not receive direct input from PRC or PHC (Naber et al., 1999, 2001; Witter et al., 2000; Furtak et al., 2007). Our findings revealed a sim- ilar pattern in human functional connectivity such that PRC showed stronger functional connectivity with anterior CA1 and subiculum, whereas PHC showed stronger functional connectivity with posterior CA1 and subiculum. Conversely, we found that this anterior- 

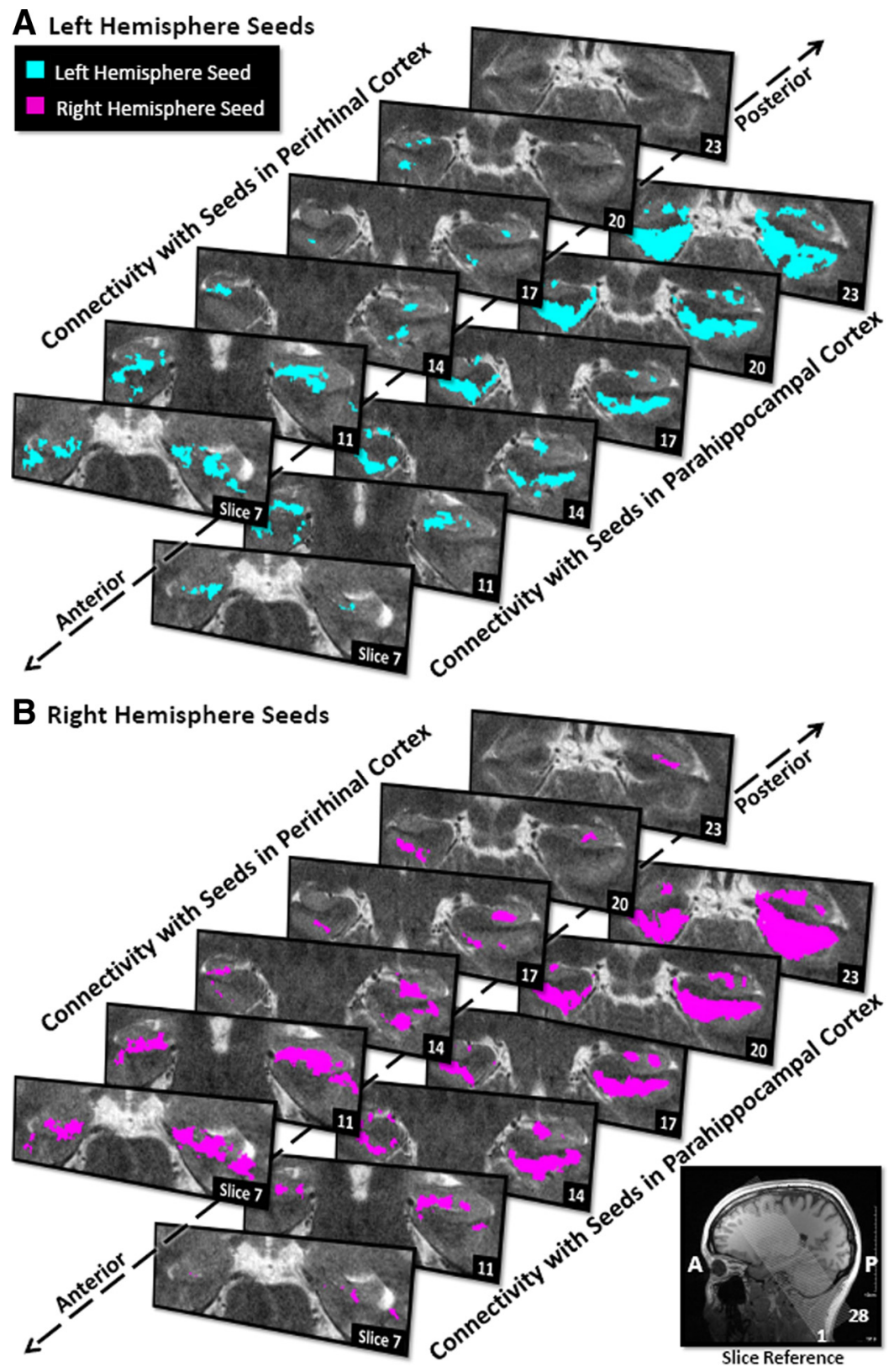

Figure 5. High-resolution analysis of hippocampal functional connectivity with PRC and PHC seed regions. $A, B$, One-sample $t$ test high-resolution functional connectivity results $\left(z>2.3, p_{\text {custer }}<0.05\right)$ mapped separately for seeds in PRC (above the diagonal) and PHC (below the diagonal). Selected slices are distributed evenly across the longitudinal axis of the hippocampus and representative of the overall spatial pattern of results. Cyan, Left hemisphere seed only; pink, right hemisphere seed only. Slices 6-12 contain HF head, PRC, and ERC; slices 13-19 contain HF body and anterior PHC; slices 20 - 25 contain HF tail and posterior PHC. The left hemisphere is on left. ferential involvement of the anterior and posterior hippocampi in episodic memory encoding and retrieval (Lepage et al., 1998), but consistent differences have not been observed (Schacter et al., 1999; Schacter and Wagner, 1999). More recent research has indicated that the human posterior hippocampus shows preferential involvement in processing of spatial or contextual information (Awipi and Davachi, 2008; Lee et al., 2008; Duarte et al., 2011; Liang et al., 2012), whereas the anterior hippocampus may be preferentially involved in memory for faces (Lee et al., 2008) and affective stimuli (Murty et al., 2010). However, this pattern has not held in all studies, as some findings suggest a domain-general role for the anterior hippocampus (Chua et al., 2007; Awipi and Davachi, 2008; Liang et al., 2012). The current results indicate that some progress toward this issue might be gained by separately considering the functional contributions of different hippocampal subfields along the longitudinal axis.

Functional connectivity with neocortex Comparison of functional connectivity profiles across the whole brain revealed that $\mathrm{PRC}$ and PHC were differentially associated with two distinct neocortical networks. PRC connected with an anterior network that included anterior lateral temporal and orbital prefrontal regions. PHC connected with a posterior cortical network that included the retrosplenial, posterior cingulate, and medial parietal cortex, as well as early visual areas in the occipital and posterior temporal cortices. These results are consistent with the major findings by Kahn et al. (2008), who reported different connectivity patterns for PRC and $\mathrm{PHC}$ in visual and visuospatial regions and medial PFC. However, we have extended PRC and PHC connectivity profiles to include additional regions implicated by animal models of anatomical connectivity, such as auditory and somatosensory areas (Burwell and Amaral, 1998a; Burwell, 2000; Agster and Burwell, 2009).

posterior connectivity gradient was less robust in CA2/CA3/DG, which could be consistent with neuroanatomical data indicating that these subfields lack direct connections with PRC or PHC.

These functional connectivity results complement findings of functional heterogeneity along the longitudinal axis of the hippocampus in both animals and humans. For instance, behavioral lesion and electrophysiological work in rats and monkeys support a role for the dorsal/posterior hippocampus in spatial memory (Colombo et al., 1998; Moser and Moser, 1998), whereas the ventral hippocampus in rodents has been implicated in anxietyrelated behaviors (for review, see Bannerman et al., 2004; Fanselow and Dong, 2010). Evidence for functional differentiation between the anterior and posterior hippocampus in humans has been more controversial; some researchers have argued for dif-
Many accounts of the functional organization of the MTL focus on relative differences between dorsal and ventral stream visual inputs to the PRC and PHC (Davachi, 2006; Eichenbaum et al., 2007; Barense et al., 2010; Diana et al., 2010; Montaldi and Mayes, 2010; Ranganath, 2010). However, the more striking pattern apparent in our results, as well as in the neuroanatomical literature, is that the PRC and PHC are differentially connected with two largely polymodal networks. For instance, in addition to visual areas, PRC connected preferentially with auditory and somatosensory regions. Furthermore, the main components of both networks are polymodal association cortices connected with PRC and PHC by major white matter tracts. The perirhinal, temporopolar, and orbitofrontal cortices correspond to a cortical network that is interconnected via the uncinate fasciculus, whereas the parahippocampal, retrosplenial, 
and medial and ventral parietal cortices correspond to a cortical network that is interconnected via the cingulum bundle (Kondo et al., 2005).

These connectivity differences may be relevant to our understanding of disease (Seeley et al., 2009). Connections of the PRC (perirhinal, anterior inferior temporal, and temporopolar cortices) are heavily affected by semantic dementia (Hodges and Patterson, 2007; Rohrer et al., 2011), whereas the posterior PHC system is less affected by this condition. In contrast, Alzheimer's disease disproportionally affects connections of the PHC (parahippocampal, retrosplenial, and medial parietal cortices), although PRC pathology is also seen (Boxer et al., 2003; Buckner et al., 2005; Rabinovici et al., 2007). Semantic dementia is typically characterized by severe deficits in knowledge about people and objects (Murre et al., 2001; Lee et al., 2007), whereas early Alzheimer's disease is associated with relatively more severe deficits in episodic memory (Nestor et al., 2006) and scene processing (Lee et al., 2007), suggesting that these two systems may support distinct behavioral functions.

Functional imaging data also suggest that the cortical networks that are differentially affiliated with the PRC and PHC have divergent functional characteristics. Whereas the PRC and ventral temporopolar cortex have been linked to familiarity-based item recognition (Brown and Aggleton, 2001; Diana et al., 2007; Eichenbaum et al., 2007), fine-grained object discriminations (Pihlajamäki et al., 2005; Lee et al., 2008; Barense et al., 2010), and access to abstract semantic knowledge (Taylor et al., 2006; Hodges and Patterson, 2007), PHC and retrosplenial function has been linked to recollection of context information (Davachi, 2006; Diana et al., 2008), simulation of episodes (Schacter et al., 2007), scene perception and imagery (Epstein, 2008; Litman et al., 2009; Vann et al., 2009; Staresina et al., 2011), and spatial navigation (Ekstrom and Bookheimer, 2007; Spreng et al., 2009; Vann et al., 2009). Put into context with evidence from brain anatomy, disease, and function, the cortical areas that show differential connectivity with PRC and PHC may be described as two distinct, polymodal, large-scale neural systems that support different forms of memory-guided behavior (Kahn et al., 2008).

\section{Limitations and future directions}

Available evidence indicates that BOLD resting-state functional connectivity corresponds well with structural connectivity, as indicated by tract tracing in animals (Vincent et al., 2007) and diffusion tensor imaging (DTI) in humans (Damoiseaux and Greicius, 2009; Greicius et al., 2009; Honey et al., 2009). BOLD resting-state functional connectivity analyses may be overly inclusive, however, such that functional connectivity is sensitive to both direct and indirect connections (Honey et al., 2009). Given that PRC and PHC are both

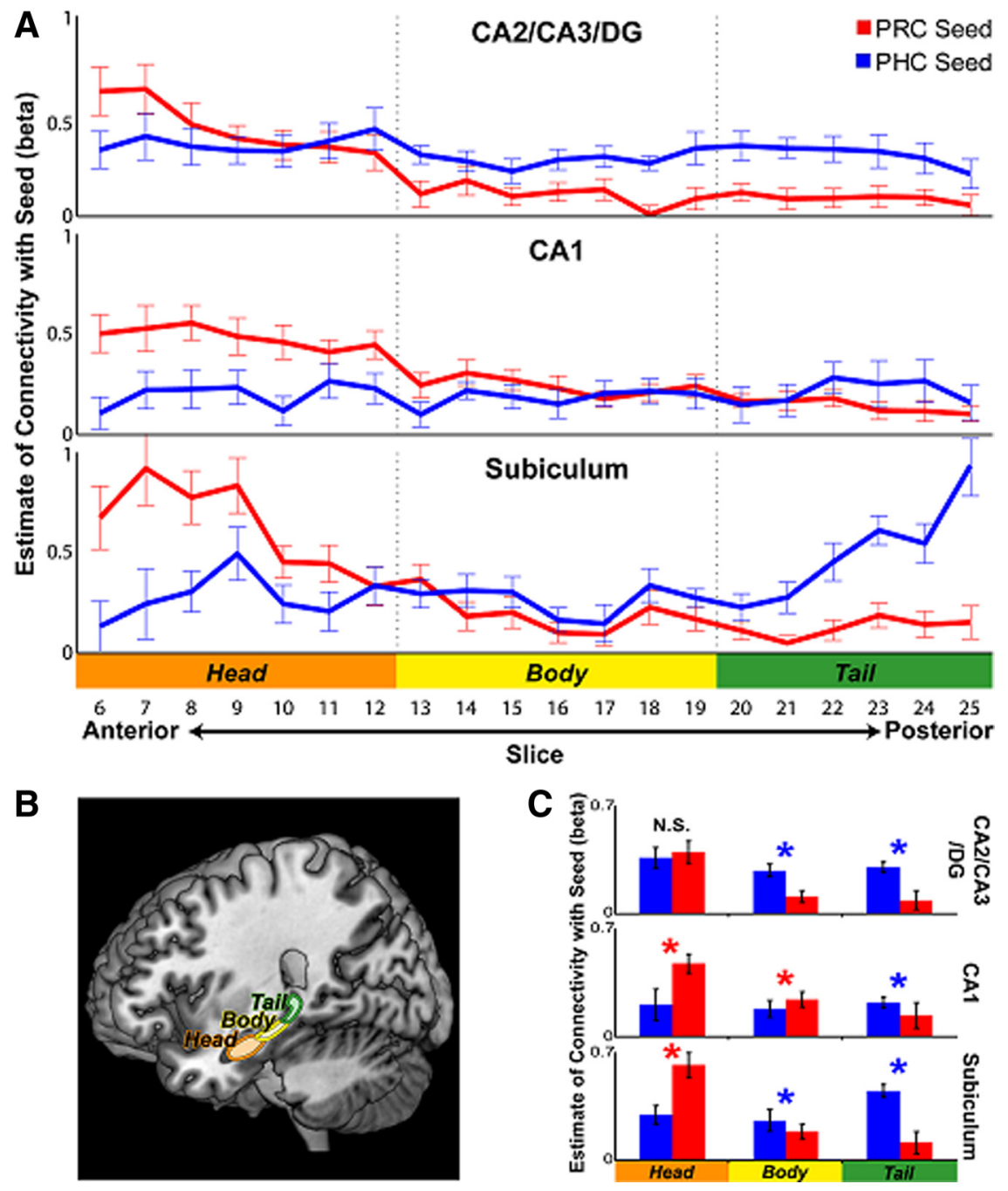

Figure 6. Functional connectivity gradients along the longitudinal hippocampal axis differ between hippocampal subfields. Red, Connectivity with PRC seed; blue, connectivity with PHC seed. Error bars denote standard error across participants. Connectivity patterns were consistent for seeds in right and left hemispheres and for extractions from right and left hippocampus; 列 ences in PRC and PHC connectivity estimate for a given segment. ${ }^{*} p<0.05$ (red asterisks, PRC connectivity greater than PHC connectivity; blue asterisks, PHC connectivity greater than PRC connectivity). N.S., Not significant.

anatomically interconnected and typically share vascular origin (Duvernoy and Bourgouin, 1998; Huther et al., 1998), one might expect this technique to reveal largely similar functional connectivity profiles for the two regions. We nonetheless observed robust differences in functional connectivity profiles of PHC and PRC that are incongruent with effects due to motion (Power et al., 2011) or vascular artifact, consistent with the view that these regions are functionally distinct (Eacott and Gaffan, 2005; Davachi, 2006; Eichenbaum et al., 2007; Lee et al., 2008; Montaldi and Mayes, 2010; Ranganath, 2010). Further insights into the anatomical bases of functional connectivity differences between PRC and PHC might be gained in future studies that combine resting-state functional connectivity analysis of fMRI data with connectivity profiles obtained from DTI data (Greicius et al., 2009).

Another issue that should be considered in future research is whether PHC and PRC show preferential connectivity with dif- 
ferent sections of ERC, as might be suggested by anatomical studies in the rat (Burwell and Amaral, 1998b) and monkey (Suzuki and Amaral, 1994a). Unfortunately, signal loss due to susceptibility artifact fundamentally limited our ability to detect effects in the ERC. Thus, an important challenge to be addressed in future resting-state fMRI and DTI studies will be to obtain highresolution data from ERC (Yassa et al., 2011).

\section{References}

Agster KL, Burwell RD (2009) Cortical efferents of the perirhinal, postrhinal, and entorhinal cortices of the rat. Hippocampus 19:1159-1186.

Awipi T, Davachi L (2008) Content-specific source encoding in the human medial temporal lobe. J Exp Psychol Learn Mem Cogn 34:769-779.

Bannerman DM, Rawlins JN, McHugh SB, Deacon RM, Yee BK, Bast T, Zhang WN, Pothuizen HH, Feldon J (2004) Regional dissociations within the hippocampus-memory and anxiety. Neurosci Biobehav Rev 28:273-283.

Barense MD, Henson RN, Lee AC, Graham KS (2010) Medial temporal lobe activity during complex discrimination of faces, objects, and scenes: effects of viewpoint. Hippocampus 20:389-401.

Barnes KA, Cohen AL, Power JD, Nelson SM, Dosenbach YB, Miezin FM, Petersen SE, Schlaggar BL (2010) Identifying Basal Ganglia divisions in individuals using resting-state functional connectivity MRI. Front Syst Neurosci 4:18.

Birn RM, Diamond JB, Smith MA, Bandettini PA (2006) Separating respiratory-variation-related fluctuations from neuronal-activity-related fluctuations in fMRI. Neuroimage 31:1536-1548.

Biswal BB, Mennes M, Zuo XN, Gohel S, Kelly C, Smith SM, Beckmann CF, Adelstein JS, Buckner RL, Colcombe S, Dogonowski AM, Ernst M, Fair D, Hampson M, Hoptman MJ, Hyde JS, Kiviniemi VJ, Kötter R, Li SJ, Lin CP, et al. (2010) Toward discovery science of human brain function. Proc Natl Acad Sci U S A 107:4734-4739.

Boxer AL, Rankin KP, Miller BL, Schuff N, Weiner M, Gorno-Tempini ML, Rosen HJ (2003) Cinguloparietal atrophy distinguishes Alzheimer disease from semantic dementia. Arch Neurol 60:949-956.

Brown MW, Aggleton JP (2001) Recognition memory: what are the roles of the perirhinal cortex and hippocampus? Nat Rev Neurosci 2:51-61.

Buckner RL, Snyder AZ, Shannon BJ, LaRossa G, Sachs R, Fotenos AF, Sheline YI, Klunk WE, Mathis CA, Morris JC, Mintun MA (2005) Molecular, structural, and functional characterization of Alzheimer's disease: evidence for a relationship between default activity, amyloid, and memory. J Neurosci 25:7709-7717.

Burwell RD (2000) The parahippocampal region: corticocortical connectivity. Ann N Y Acad Sci 911:25-42.

Burwell RD, Amaral DG (1998a) Cortical afferents of the perirhinal, postrhinal, and entorhinal cortices of the rat. J Comp Neurol 398:179-205.

Burwell RD, Amaral DG (1998b) Perirhinal and postrhinal cortices of the rat: interconnectivity and connections with the entorhinal cortex. J Comp Neurol 391:293-321.

Chua EF, Schacter DL, Rand-Giovannetti E, Sperling RA (2007) Evidence for a specific role of the anterior hippocampal region in successful associative encoding. Hippocampus 17:1071-1080.

Colombo M, Fernandez T, Nakamura K, Gross CG (1998) Functional differentiation along the anterior-posterior axis of the hippocampus in monkeys. J Neurophysiol 80:1002-1005.

Damoiseaux JS, Greicius MD (2009) Greater than the sum of its parts: a review of studies combining structural connectivity and resting-state functional connectivity. Brain Struct Funct 213:525-533.

Davachi L (2006) Item, context and relational episodic encoding in humans. Curr Opin Neurobiol 16:693-700.

Diana RA, Yonelinas AP, Ranganath C (2007) Imaging recollection and familiarity in the medial temporal lobe: a three-component model. Trends Cogn Sci 11:379-386.

Diana RA, Yonelinas AP, Ranganath C (2008) High-resolution multi-voxel pattern analysis of category selectivity in the medial temporal lobes. Hippocampus 18:536-541.

Diana RA, Yonelinas AP, Ranganath C (2010) Medial temporal lobe activity during source retrieval reflects information type, not memory strength. J Cogn Neurosci 22:1808-1818.

Duarte A, Henson RN, Graham KS (2011) Stimulus content and the neural correlates of source memory. Brain Res 1373:110-123.

Dudukovic NM, Preston AR, Archie JJ, Glover GH, Wagner AD (2011)
High-resolution fMRI reveals match enhancement and attentional modulation in the human medial temporal lobe. J Cogn Neurosci 23:670-682.

Duvernoy HM, Bourgouin P (1998) The human hippocampus: functional anatomy, vascularization and serial sections with MRI, Ed 2. New York: Springer.

Eacott MJ, Gaffan EA (2005) The roles of perirhinal cortex, postrhinal cortex, and the fornix in memory for objects, contexts, and events in the rat. Q J Exp Psychol B 58:202-217.

Eichenbaum H (2006) Remembering: functional organization of the declarative memory system. Curr Biol 16:R643-R645.

Eichenbaum H, Yonelinas AP, Ranganath C (2007) The medial temporal lobe and recognition memory. Annu Rev Neurosci 30:123-152.

Ekstrom AD, Bookheimer SY (2007) Spatial and temporal episodic memory retrieval recruit dissociable functional networks in the human brain. Learn Mem 14:645-654.

Ekstrom AD, Bazih AJ, Suthana NA, Al-Hakim R, Ogura K, Zeineh M, Burggren AC, Bookheimer SY (2009) Advances in high-resolution imaging and computational unfolding of the human hippocampus. Neuroimage 47:42-49.

Epstein RA (2008) Parahippocampal and retrosplenial contributions to human spatial navigation. Trends Cogn Sci 12:388-396.

Erdem A, Yaşargil G, Roth P (1993) Microsurgical anatomy of the hippocampal arteries. J Neurosurg 79:256-265.

Fanselow MS, Dong HW (2010) Are the dorsal and ventral hippocampus functionally distinct structures? Neuron 65:7-19.

Firbank MJ, Harrison RM, Williams ED, Coulthard A (2000) Quality assurance for MRI: practical experience. Br J Radiol 73:376-383.

Furtak SC, Wei SM, Agster KL, Burwell RD (2007) Functional neuroanatomy of the parahippocampal region in the rat: the perirhinal and postrhinal cortices. Hippocampus 17:709-722.

Greicius MD, Supekar K, Menon V, Dougherty RF (2009) Resting-state functional connectivity reflects structural connectivity in the default mode network. Cereb Cortex 19:72-78.

Hodges JR, Patterson K (2007) Semantic dementia: a unique clinicopathological syndrome. Lancet Neurol 6:1004-1014.

Honey CJ, Sporns O, Cammoun L, Gigandet X, Thiran JP, Meuli R, Hagmann P (2009) Predicting human resting-state functional connectivity from structural connectivity. Proc Natl Acad Sci U S A 106:2035-2040.

Huther G, Dörfl J, Van der Loos H, Jeanmonod D (1998) Microanatomic and vascular aspects of the temporomesial region. Neurosurgery 43:1118-1136.

Huynh H, Feldt LS (1976) Estimation of the box correction for degrees of freedom from sample data in randomized block and split-plot designs. J Educ Stat 1:69-82.

Insausti R, Juottonen K, Soininen $\mathrm{H}$, Insausti AM, Partanen K, Vainio P, Laakso MP, Pitkänen A (1998) MR volumetric analysis of the human entorhinal, perirhinal, and temporopolar cortices. Am J Neuroradiol 19:659-671.

Jenkinson M, Smith S (2001) A global optimisation method for robust affine registration of brain images. Med Image Anal 5:143-156.

Jenkinson M, Bannister P, Brady M, Smith S (2002) Improved optimization for the robust and accurate linear registration and motion correction of brain images. Neuroimage 17:825-841.

Kahn I, Andrews-Hanna JR, Vincent JL, Snyder AZ, Buckner RL (2008) Distinct cortical anatomy linked to subregions of the medial temporal lobe revealed by intrinsic functional connectivity. J Neurophysiol 100:129-139.

Kerr KM, Agster KL, Furtak SC, Burwell RD (2007) Functional neuroanatomy of the parahippocampal region: the lateral and medial entorhinal areas. Hippocampus 17:697-708.

Kondo H, Saleem KS, Price JL (2005) Differential connections of the perirhinal and parahippocampal cortex with the orbital and medial prefrontal networks in macaque monkeys. J Comp Neurol 493:479-509.

Lavenex P, Amaral DG (2000) Hippocampal-neocortical interaction: a hierarchy of associativity. Hippocampus 10:420-430.

Lecoutre B (1991) A correction for the $\tilde{\varepsilon}$ approximate test in repeated measures designs with two or more independent groups. J Educ Stat 16:371-372.

Lee AC, Levi N, Davies RR, Hodges JR, Graham KS (2007) Differing profiles of face and scene discrimination deficits in semantic dementia and Alzheimer's disease. Neuropsychologia 45:2135-2146.

Lee AC, Scahill VL, Graham KS (2008) Activating the medial temporal lobe during oddity judgment for faces and scenes. Cereb Cortex 18:683-696. 
Lepage M, Habib R, Tulving E (1998) Hippocampal PET activations of memory encoding and retrieval: the HIPER model. Hippocampus 8:313-322.

Liang JC, Wagner AD, Preston AR (2012) Content representation in the human medial temporal lobe. Cereb Cortex. Advance online publication. Retrieved January 23, 2012. doi:10.1093/cercor/bhr379.

Litman L, Awipi T, Davachi L (2009) Category-specificity in the human medial temporal lobe cortex. Hippocampus 19:308-319.

Lorente de No R (1934) Studies of the structure of the cerebral cortex. The continuation of the study of the ammonic system. J Psychol Neurol 46:113-117.

Marinković SV, Milisavljević MM, Vucković VD (1991) Microvascular anatomy of the uncus and the parahippocampal gyrus. Neurosurgery 29:805-814.

Marinković S, Milisavljević M, Puskas L (1992) Microvascular anatomy of the hippocampal formation. Surg Neurol 37:339-349.

Montaldi D, Mayes AR (2010) The role of recollection and familiarity in the functional differentiation of the medial temporal lobes. Hippocampus 20:1291-1314.

Moser MB, Moser EI (1998) Functional differentiation in the hippocampus. Hippocampus 8:608-619.

Murre JM, Graham KS, Hodges JR (2001) Semantic dementia: relevance to connectionist models of long-term memory. Brain 124:647-675.

Murty VP, Ritchey M, Adcock RA, LaBar KS (2010) fMRI studies of successful emotional memory encoding: a quantitative meta-analysis. Neuropsychologia 48:3459-3469.

Naber PA, Witter MP, Lopez da Silva FH (1999) Perirhinal cortex input to the hippocampus in the rat: evidence for parallel pathways, both direct and indirect. A combined physiological and anatomical study. Eur J Neurosci 11:4119-4133.

Naber PA, Witter MP, Lopes da Silva FH (2001) Evidence for a direct projection from the postrhinal cortex to the subiculum in the rat. Hippocampus 11:105-117.

Nestor PJ, Fryer TD, Hodges JR (2006) Declarative memory impairments in Alzheimer's disease and semantic dementia. Neuroimage 30:1010-1020.

Pihlajamäki M, Tanila H, Könönen M, Hänninen T, Aronen HJ, Soininen H (2005) Distinct and overlapping fMRI activation networks for processing of novel identities and locations of objects. Eur J Neurosci 22:2095-2105.

Power JD, Barnes KA, Snyder AZ, Schlaggar BL, Petersen SE (2011) Spurious but systematic correlations in functional connectivity MRI networks arise from subject motion. Neuroimage 59:2142-2154.

Rabinovici GD, Seeley WW, Kim EJ, Gorno-Tempini ML, Rascovsky K, Pagliaro TA, Allison SC, Halabi C, Kramer JH, Johnson JK, Weiner MW, Forman MS, Trojanowski JQ, Dearmond SJ, Miller BL, Rosen HJ (2007) Distinct MRI atrophy patterns in autopsy-proven Alzheimer's disease and frontotemporal lobar degeneration. Am J Alzheimers Dis Other Demen 22:474-488.

Raichle ME, MacLeod AM, Snyder AZ, Powers WJ, Gusnard DA, Shulman GL (2001) A default mode of brain function. Proc Natl Acad Sci U S A 98:676-682.

Ranganath C (2010) A unified framework for the functional organization of the medial temporal lobes and the phenomenology of episodic memory. Hippocampus 20:1263-1290.

Rohrer JD, Lashley T, Schott JM, Warren JE, Mead S, Isaacs AM, Beck J, Hardy J, de Silva R, Warrington E, Troakes C, Al-Sarraj S, King A, Borroni B, Clarkson MJ, Ourselin S, Holton JL, Fox NC, Revesz T, Rossor MN, et al. (2011) Clinical and neuroanatomical signatures of tissue pathology in frontotemporal lobar degeneration. Brain 134:2565-2581.
Schacter DL, Wagner AD (1999) Medial temporal lobe activations in fMRI and PET studies of episodic encoding and retrieval. Hippocampus 9:7-24.

Schacter DL, Curran T, Reiman EM, Chen K, Bandy DJ, Frost JT (1999) Medial temporal lobe activation during episodic encoding and retrieval: a PET study. Hippocampus 9:575-581.

Schacter DL, Addis DR, Buckner RL (2007) Remembering the past to imagine the future: the prospective brain. Nat Rev Neurosci 8:657-661.

Seeley WW, Crawford RK, Zhou J, Miller BL, Greicius MD (2009) Neurodegenerative diseases target large-scale human brain networks. Neuron 62:42-52.

Skudlarski P, Jagannathan K, Calhoun VD, Hampson M, Skudlarska BA, Pearlson G (2008) Measuring brain connectivity: diffusion tensor imaging validates resting state temporal correlations. Neuroimage 43:554561.

Spreng RN, Mar RA, Kim AS (2009) The common neural basis of autobiographical memory, prospection, navigation, theory of mind, and the default mode: a quantitative meta-analysis. J Cogn Neurosci 21:489-510.

Squire LR, Zola-Morgan S (1991) The medial temporal lobe memory system. Science 253:1380-1386.

Staresina BP, Duncan KD, Davachi L (2011) Perirhinal and parahippocampal cortices differentially contribute to later recollection of object- and scene-related event details. J Neurosci 31:8739-8747.

Suthana N, Ekstrom A, Moshirvaziri S, Knowlton B, Bookheimer S (2010) Dissociations within human hippocampal subregions during encoding and retrieval of spatial information. Hippocampus 21:694-701.

Suzuki WA, Amaral DG (1994a) Topographic organization of the reciprocal connections between the monkey entorhinal cortex and the perirhinal and parahippocampal cortices. J Neurosci 14:1856-1877.

Suzuki WA, Amaral DG (1994b) Perirhinal and parahippocampal cortices of the macaque monkey: cortical afferents. J Comp Neurol 350:497-533.

Taylor KI, Moss HE, Stamatakis EA, Tyler LK (2006) Binding crossmodal object features in perirhinal cortex. Proc Natl Acad Sci USA 103:8239-8244.

van den Heuvel MP, Hulshoff Pol HE (2010) Exploring the brain network: a review on resting-state fMRI functional connectivity. Eur Neuropsychopharmacol 20:519-534.

Vann SD, Aggleton JP, Maguire EA (2009) What does the retrosplenial cortex do? Nat Rev Neurosci 10:792-802.

Vincent JL, Patel GH, Fox MD, Snyder AZ, Baker JT, Van Essen DC, Zempel JM, Snyder LH, Corbetta M, Raichle ME (2007) Intrinsic functional architecture in the anaesthetized monkey brain. Nature 447:83-86.

Wise RG, Ide K, Poulin MJ, Tracey I (2004) Resting fluctuations in arterial carbon dioxide induce significant low frequency variations in BOLD signal. Neuroimage 21:1652-1664.

Witter MP, Wouterlood FG, Naber PA, Van Haeften T (2000) Anatomical organization of the parahippocampal-hippocampal network. Ann N Y Acad Sci 911:1-24.

Worsley KJ, Marrett S, Neelin P, Vandal AC, Friston KJ, Evans AC (1996) A unified statistical approach for determining significant signals in images of cerebral activation. Hum Brain Mapp 4:58-73.

Yassa MA, Stark CE (2009) A quantitative evaluation of cross-participant registration techniques for MRI studies of the medial temporal lobe. NeuroImage 44:319-327.

Yassa MA, Mattfeld AT, Stark SM, Stark CE (2011) Age-related memory deficits linked to circuit-specific disruptions in the hippocampus. Proc Natl Acad Sci U S A 108:8873-8878.

Zeineh MM, Engel SA, Thompson PM, Bookheimer SY (2001) Unfolding the human hippocampus with high resolution structural and functional MRI. Anat Rec 265:111-120. 